\title{
4-Axis printing microfibrous tubular scaffold and tracheal cartilage application
}

\author{
Dong Lei ${ }^{1,2}$, Bin Luo $^{2}$, Yifan Guo ${ }^{2}$, Di Wang ${ }^{4}$, Hao Yang ${ }^{4}$, Shaofei Wang ${ }^{2}$, Huixia Xuan ${ }^{2}$, Ao Shen ${ }^{2}$, \\ Yi Zhang ${ }^{2}$, Zenghe Liu ${ }^{1,2}$, Chuanglong $\mathrm{He}^{1}$, Feng-Ling Qing ${ }^{1}$, Yong $\mathrm{Xu}^{3^{*}}$, Guangdong Zhou ${ }^{5,6^{*}}$ and \\ Zhengwei You ${ }^{1,2^{*}}$
}

\begin{abstract}
Long-segment defects remain a major problem in clinical treatment of tubular tissue reconstruction. The design of tubular scaffold with desired structure and functional properties suitable for tubular tissue regeneration remains a great challenge in regenerative medicine. Here, we present a reliable method to rapidly fabricate tissueengineered tubular scaffold with hierarchical structure via 4axis printing system. The fabrication process can be adapted to various biomaterials including hydrogels, thermoplastic materials and thermosetting materials. Using polycaprolactone (PCL) as an example, we successfully fabricated the scaffolds with tunable tubular architecture, controllable mesh structure, radial elasticity, good flexibility, and luminal patency. As a preliminary demonstration of the applications of this technology, we prepared a hybrid tubular scaffold via the combination of the 4-axis printed elastic poly(glycerol sebacate) (PGS) bio-spring and electrospun gelatin nanofibers. The scaffolds seeded with chondrocytes formed tubular mature cartilage-like tissue both via in vitro culture and subcutaneous implantation in the nude mouse, which showed great potential for tracheal cartilage reconstruction.
\end{abstract}

Keywords: 3D printing, tissue engineering, tubular scaffold, tracheal cartilage

\section{INTRODUCTION}

Tubular tissues such as blood vessel, trachea, and urethra are important organs in human body. Cancer and trauma often result in their diseases and long-segment defects [1]. The demand for tubular tissue reconstruction is large in clinical treatment [2]. At present, most tube substitutes in clinic are weaved by Dacron ${ }^{\mathrm{TM}}$ or expanded polytetrafluoroethylene (ePTFE) [3]. However, as alternative foreign bodies are implanted in the body for a long time, these kinds of grafts lack biological activity. Tissue engineering represents a promising solution for the functional reconstruction of tubular organs such as the blood vessel, trachea, gastrointestinal tract, and urinary tract [47]. Tubular organs are characterized by complex structure and mechanical properties that are responsible for specific functions [8,9]. Development in tissue engineering in recent years and promising results, showed an urgent need for investigating more complex constructs that are designed in special and accurate manner [10-12]. This brings additional challenges in tissue engineering in order to construct complete organs by using combinations of various cells along with the support material systems.

Numerous attempts have been made to develop functional tubular scaffolds in regenerative medicine $[13,14]$. The mold method is a universal approach to process polymeric biomaterials into synthetic tubular scaffold [15]. But this method involves the complicated multi-step processing and inconvenient structural adjustment. Electrospinning is an effective way to fabricate nanofibrous tubular scaffold with tailorable and suitable characteristics for the appropriate function as hollow

${ }^{1}$ State Key Laboratory for Modification of Chemical Fibers and Polymer Materials, College of Chemistry, Chemical Engineering and Biotechnology, Donghua University, Shanghai 201620, China

${ }^{2}$ Shanghai Belt and Road Joint Laboratory of Advanced Fiber and Low-dimension Materials (Donghua University), College of Materials Science and Engineering, Donghua University, Shanghai 201620, China

${ }^{3}$ Department of Thoracic Surgery, Shanghai Pulmonary Hospital, Tongji University School of Medicine, Shanghai 200433, China

${ }^{4}$ Department of Mechanical Engineering and Automation, Shanghai University, Shanghai 200072, China

${ }^{5}$ Shanghai Key Lab of Tissue Engineering, Shanghai 9th People’s Hospital, Shanghai Jiao Tong University School of Medicine, Shanghai 200011, China

${ }^{6}$ Research Institute of Plastic Surgery, Weifang Medical University, Weifang 261053, China

* Corresponding authors (emails: xuyong103300@163.com (Xu Y); guangdongzhou@126.com (Zhou G); zyou@dhu.edu.cn (You Z)) 
organ, but this method has also time-consuming problems due to low productive efficiency of nanofibers [4]. The preparation of a hollow scaffold may require a couple of hours. Moreover, the electrospinning method mainly applies to plastic materials $[16,17]$. Therefore, it is necessary to develop a versatile method to process various biomaterials into tubular scaffold with controllable and adjustable morphostructure.

Recently, extrusion-based three-dimensional (3D) printing technology has been widely applied for producing biomaterials in regenerative medicine and promoted significant innovations [18-22]. During printing, viscoelastic inks are extruded out of a 3D printer's nozzle as printed fibers, which are deposited into patterns layer by layer when the nozzle moves $[23,24]$. It is difficult to fabricate porous and thin-walled tubular scaffold because the printed fibers lack the buttressing effect [25]. Robertson et al. [19] demonstrated that viscous ink was extruded from a print head and frontally polymerized immediately upon exiting the nozzle, allowing for the simultaneous free-form printing to fabricate spiral structure. However, this method only applies to specific material.

Here, we report a simple strategy to rapidly fabricate microfibrous tubular scaffold with braid structure using a 4 -axis printing system. This approach is highly versatile and readily applies to diverse materials including thermoplastic materials, thermosets and hydrogels. The tubular scaffolds with various macroscopic morphologies and microstructures could be readily tailored by controlling related parameters. To demonstrate the utilization, we fabricated a hybrid tubular scaffold via the combination of the 4-axis printed elastic bio-spring and electrospun nanofibers. When combined with chondrocytes, this hybrid tubular scaffold could regenerate satisfactory tubular cartilage with similar strength and elasticity to native tracheal cartilage both in vitro and in vivo.

\section{EXPERIMENTAL SECTION}

\section{Materials}

Polycaprolactone (PCL, $M_{\mathrm{n}}=80,000 \mathrm{~g} \mathrm{~mol}^{-1}$ ), sodium alginate, calcium chloride dehydrate $(\geqslant 99 \%)$, ammonium persulfate $(\geqslant 98 \%)$ and gelatin from porcine skin (Type A) were purchased from Sigma-Aldrich (USA). Glycerol (analytical grade, $\geqslant 99 \%$ ), sebacic acid (analytical grade, $\geqslant 99 \%$ ), acrylamide (99\%) and methylene-bisacrylamide (98\%) were purchased from J\&K Scientific Ltd. (China). Sodium chloride $(\geqslant 99.5 \%)$ was purchased from Shanghai
Lingfeng Chemical Reagent Co., Ltd. (China).

\section{4-Axis printing PCL tubular scaffolds}

The PCL tubular scaffolds were fabricated using a 4-axis printing system, which consisted of direct ink writing (DIW) 3D printer (HTS-400; Fochif Mechatronics Technology, Co., China) and a rotary receiver. PCL was preheated to $200^{\circ} \mathrm{C}$ for $30 \mathrm{~min}$ in the extruder and melted completely. Then the melted PCL was squeezed out into filaments at $180^{\circ} \mathrm{C}$. Extrusion rate was $0.03 \mathrm{~mm} / \mathrm{min}$. Extruder internal diameter was $18 \mathrm{~mm}$. Nozzle size was 22G. The extrusion nozzle moved along the central shaft of receiver at $1 \mathrm{~mm} / \mathrm{s}$. Several types of tubular scaffolds were fabricated using special-shaped receivers (triangular prism, hexagonal prism and cone frustum) and cylindrical receivers with different diameters $(2,5$ and $10 \mathrm{~mm})$. The receiver rotated constantly at 20 revolutions per minute $(n=20)$. In order to obtain scaffolds with controllable microstructure, different rotary rates of receiver were investigated $(n=10,20,30$ and 40$)$. Other printing parameters remained unchanged as follows: extrusion rate $\left(V_{\mathrm{e}}\right)$ was $0.03 \mathrm{~mm} / \mathrm{min}$; nozzle-moving rate $\left(V_{\mathrm{m}}\right)$ was $1 \mathrm{~mm} / \mathrm{s}$; extruder diameter $\left(D_{\mathrm{e}}\right)$ was $18 \mathrm{~mm}$; diameter of cylindrical receiver $\left(D_{\mathrm{r}}\right)$ was $5.14 \mathrm{~mm}$. The printer kept reciprocating movement for 5 times.

\section{4-Axis printing hydrogel tubular scaffolds}

Sodium alginate $(0.6 \mathrm{~g})$, acrylamide $(4 \mathrm{~g})$ and methylenebisacrylamide $(3.6 \mathrm{mg})$ were dissolved and mixed together in deionized water $(10 \mathrm{~mL})$ to form $3 \mathrm{D}$ printable viscoelastic ink. Ammonium persulfate was added in $2 \mathrm{wt} \%$ of the acrylamide before printing. For images in the figures, red pigment for hydrogel was added in $1 \%$ $(w / v)$ of the total hydrogel ink. The ink was extruded into fiber and then deposited on the rotational cylindrical receiver. $\mathrm{CaCl}_{2}$ aqueous solution $(10 \%, w / v)$ was sprayed onto the hydrogel fiber to induce the gelation of sodium alginate with divalent cation during the printing process. Then the printed construct was polymerized to form the tubular hydrogel scaffold under UV irradiation with wavelength of $365 \mathrm{~nm}$ for $5 \mathrm{~min}$.

\section{4-Axis printing thermoset PGS bio-spring}

Poly(glycerol sebacate) (PGS) pre-polymer was synthesized through the polycondensation of equimolar amounts of glycerol and sebacic acid at $120^{\circ} \mathrm{C}$ and under nitrogen atmosphere for $72 \mathrm{~h}$ according to the previous report [26]. Melt PGS prepolymer was mixed with salt particles $(\mathrm{NaCl}, 38-76 \mu \mathrm{m})$ at $50^{\circ} \mathrm{C}$ in a weight ratio of $1: 2$ to prepare printable ink. The composite ink was extruded 
into homogeneous filament, which was continuously deposited on the rotational cylindrical receiver (with a diameter of $5.14 \mathrm{~mm}$ ). The printed construct with receiver was transferred to a vacuum oven and cured at $100^{\circ} \mathrm{C}(0.5 \mathrm{bar})$ for $12 \mathrm{~h}$ and then further cured at $150^{\circ} \mathrm{C}$ (1 bar) for $24 \mathrm{~h}$. After curing, the resultant constructs were soaked in distilled water to remove the salt for $24 \mathrm{~h}$, with the water changed three times. Finally, the highly porous PGS tubular bio-spring was freeze-dried in vacuum.

\section{Fabrication of hybrid tubular scaffold}

Gelatin was electrospun outside of the PGS bio-spring to produce the bilayered tubular scaffold. A suitable steel mandrel (with a diameter of $4 \mathrm{~mm}$ ) was inserted into the lumen of PGS bio-spring. Then, the steel mandrel was fixed in the rotatable collector, which was placed at a distance of $15 \mathrm{~cm}$ from the needle tip and rotated at $100 \mathrm{r} / \mathrm{min}$. Next, a $15 \%(w / v)$ gelatin solution in hexafluoroisopropanol was pumped out at a flow rate of $1 \mathrm{~mL} / \mathrm{h}$ with a high positive voltage of $12 \mathrm{kV}$ applied. The electrospinning process lasted $1 \mathrm{~h}$ to produce dense nanofibrous gelatin layer outside of the PGS bio-spring.

\section{Characterization of tubular scaffolds}

To illustrate the multi-level morphology, PCL tubular scaffolds, PGS bio-spring and PGS/gelatin hybrid scaffolds were investigated through scanning electron microscopy (SEM; JSM-5600LV, Jeol, Ltd., Japan) under different magnification in top view and side view. The scaffolds were mounted on aluminum stubs that were sputtered with gold before analysis. The scaffolds were cut with a sharp knife to transversely reveal the cross section of lumen. To investigate filaments of the 3D printed PCL tubular scaffolds and micropores of the PGS bio-spring, SEM image data were analyzed to evaluate the filaments and micropores features by using the NIH ImageJ version 1.51. The mechanical properties of the $3 \mathrm{D}$ printed tubular scaffolds were evaluated using a universal testing machine equipped with a $100 \mathrm{~N}$ sensor (MTS Echo, Exceed 40, USA) that was equipped with the software TestSuite TW. Each PCL scaffold ( $n=10,20,30$ and 40) was compressed to a strain of $40 \%$ and stretched to a strain of $80 \%$ for 10 cycles at a rate of $10 \mathrm{~mm} / \mathrm{min}$ in compressive and tensile fatigue test, respectively. The PGS bio-spring was compressed to strains of $50 \%$ with loading-unloading for 50 cycles at a rate of $10 \mathrm{~mm} / \mathrm{min}$.

Tubular cartilage reconstruction of hybrid tubular scaffold This project was approved by the Shanghai Pulmonary
Hospital Ethics Committee. The hybrid tubular scaffolds were disinfected with $75 \%$ ethanol solution for $60 \mathrm{~min}$ and washed thrice with phosphate buffer saline. Chondrocytes was isolated from auricular cartilage of New Zealand white rabbits as previous report [27]. The harvested chondrocytes (in passage 2) were resuspended in a Dulbecco's modified Eagle medium (DMEM, Gibco) containing 10\% fetal bovine serum (FBS; Hyclone, USA) to a final concentration of $1.0 \times 10^{8}$ cells $/ \mathrm{mL}$. The suspension was seeded evenly onto the hybrid tubular scaffolds to form cell-scaffold constructs $(150 \mu \mathrm{L}$ in each scaffold). All constructs (a total of 10 samples) were incubated for $4 \mathrm{~h}$ at $37^{\circ} \mathrm{C}$ and then cultured in DMEM containing $10 \% \mathrm{FBS}$ at $37^{\circ} \mathrm{C}$ in $5 \% \mathrm{CO}_{2}$. After 2 weeks of cultivation, the constructs were either maintained in vitro for another 6 weeks or subcutaneously implanted into nude mice for 12 weeks. Scaffolds alone were implanted likewise as a control $(n=5)$. All in vitro and in vivo samples ( $n=5$ per group) were harvested for cartilage regeneration evaluation.

\section{Biocompatibility of the hybrid scaffold}

Chondrocyte viability in the scaffolds was determined at day 1 and day 4 using the Live and Dead Cell Viability Assay (Invitrogen, USA) and examined by confocal microscope (Nikon, A1RMP, Japan). Chondrocyte apoptosis in the scaffold was examined by apoptosis tunel kit (Roche, China, 11684817910) according to the manufacturer's protocol. Cell proliferation was determined using a total DNA quantification assay. The chondrocytescaffold constructs were cultured for 1 and $4 \mathrm{~d}$. Chondrocytes cultured in DMEM with 10\% FBS only (without scaffold) were used as control group.

\section{Histological and immunohistochemical analyses}

Samples of both in vitro and in vivo neocartilage were fixed in $4 \%$ paraformaldehyde, embedded in paraffin and sectioned. Sections were stained according to previously established methods with haematoxylin and eosin (HE), Safranin-O, Masson's trichrome to evaluate histological structure and cartilage extracellular matrix (ECM) deposition in the regenerated tracheal cartilage. For immunohistochemical analysis, expression of type II collagen was detected to further confirm a cartilagespecific phenotype, as described previously [28].

\section{Quantitative analyses}

All samples of three different groups, including scaffold, scaffold \& cell, and normal trachea cartilage, were cut into the same square shape $(0.5 \mathrm{~cm} \times 0.5 \mathrm{~cm})$ for the following 
quantitative analyses.

Young's modulus was determined using a biomechanical analyzer (Instron-5542, Canton, USA) as previously described. All samples from different groups (scaffold, scaffold \& cell, and normal trachea cartilage, $n=5$ per group) were applied with a continuous planar unconfined strain rate of $1 \mathrm{~mm} / \mathrm{min}$ until $80 \%$ of maximal deformation was achieved. The Young's moduli of tested samples were calculated based on the slope of the stress-strain curve. All samples from different groups were weighed with an electronic balance and their thickness was measured with a vernier caliper. Samples from three different groups ( $n=5$ per group) were digested in papain solution (Sigma-Aldrich, USA) at $65^{\circ} \mathrm{C}$. Sulfated glycosaminoglycan (GAG) contents were quantified by an Alcian Blue method. Genomic DNA was recovered in elution buffer after ethanol extraction and column adsorption. DNA content was detected using a nucleic acid protein quantitation detector (Nanodrop 2000, ThermoFisher Scientific, USA). Analysis of each sample was repeated three times. Content of total collagen in different groups was quantified by a hydroxyproline assay. Samples were prepared by alkaline hydrolysis and free hydro-xyproline hydrolyzates were assayed according to a previously described method [29].

\section{Statistical analysis}

All quantitative data are shown as means \pm standard deviation. Upon confirmation of normal data distribution, one-way analysis of variance and the posthoc least significant difference test were used to determine statistical significance among groups.

\section{RESULTS AND DISCUSSION}

4-Axis printing tubular scaffolds with diverse geometries The schematic of 4 -axis printing system is shown in Fig. 1. This is a versatile approach that can be adapted to various polymers including thermoplastics, thermosets and hydrogels to fabricate tubular scaffold with wellorganized structures and diverse properties for a wide range of tubular organs applications. Here, as a proof of principle, we first investigated the 4-axis printing of a widely used thermoplastic biomaterial PCL which has been approved by FDA for clinical applications (e.g., drug delivery system).

As shown in the photographic image in Fig. 2a, b, PCL tubular scaffolds were fabricated with different lumen diameters. The tube walls were weaved by well-organized fibers in vertical and sectional views of SEM images (Fig. 2c, d). Stable braided structure could maintain the original tubular structure without collapsing. Furthermore, this method could be used to readily construct hollow scaffolds with diverse geometries such as triangular prism (Fig. 2e-h), hexagonal prism (Fig. 2i-l) and cone frustum (Fig. S1) by changing the shape of receivers.

\section{4-Axis printing PCL tubular scaffolds with controllable microstructure and tunable mechanical properties}

Based on this 4-axis printing method, we used PCL as the representative biomaterial to create tubular scaffolds and further investigated their microstructures. As shown in Fig. 3a, hot-melt polymer was extruded out and deposited on the surface of receiver. With the cooperative work of DIW 3D printer and cylindrical receiver, the fiber was spirally arranged. Thermal extrusion and hot drawing provided an effective way to fabricate filamentous PCL, the diameter of which was smaller than printing nozzle. The periodic PCL fibers were bound solidly together in upper and lower layers to form a stable network by the reciprocating motion of printer. The molten state of polymer facilitated the formation of smooth melt fusions at filament intersections. The blue filaments represented the segments of a same spiral fiber (Fig. 3b). By varying the rotational rate of receiver $(n=10,20,30,40 \mathrm{r} / \mathrm{min})$, while holding other parameters constant, we fabricated

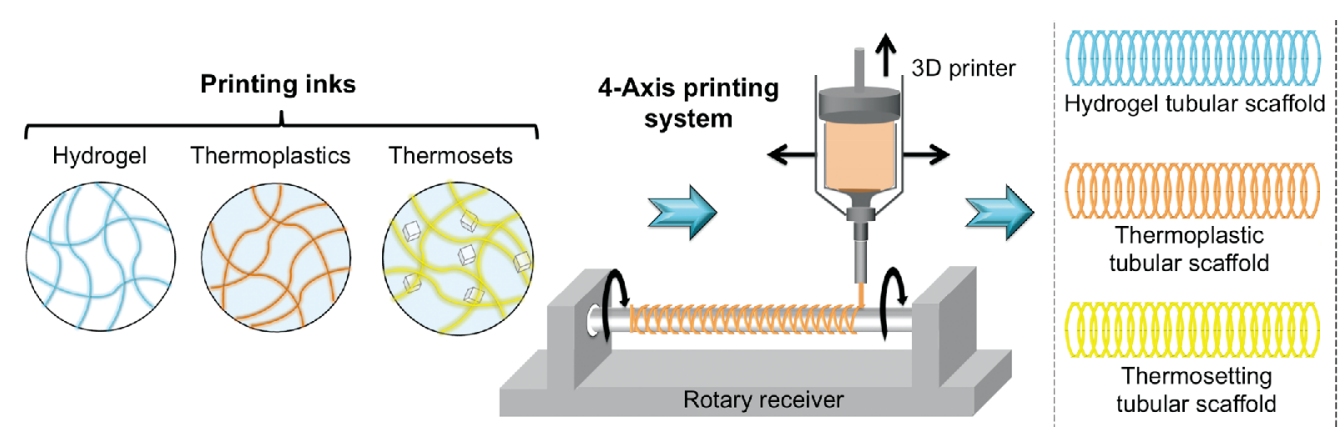

Figure 1 Schematic illustration of the 4-axis printing system to prepare tubular scaffolds from various materials. 

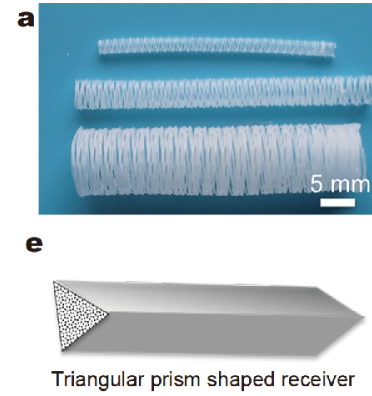

i

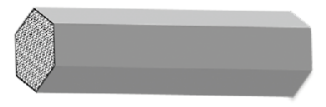

Hexagonal prism shaped receiver
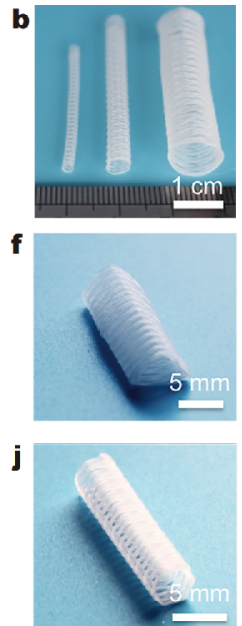
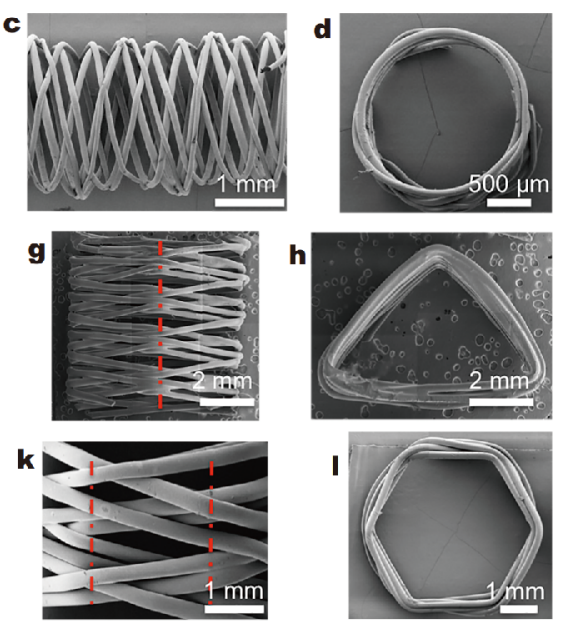

Figure 2 Printed PCL tubular scaffolds with diverse geometries. Tubular scaffolds with different diameters (a, b); SEMs of circular tubular scaffold in top view (c) and section view (d); triangular prism receiver (e) and corresponding tubular scaffold (f); SEMs of triangular tubular scaffold in top view (g) and section view (h); hexagonal prism shaped receiver (i) and corresponding tubular scaffold (j); SEMs of hexagonal tubular scaffold in top view (k) and section view (l).
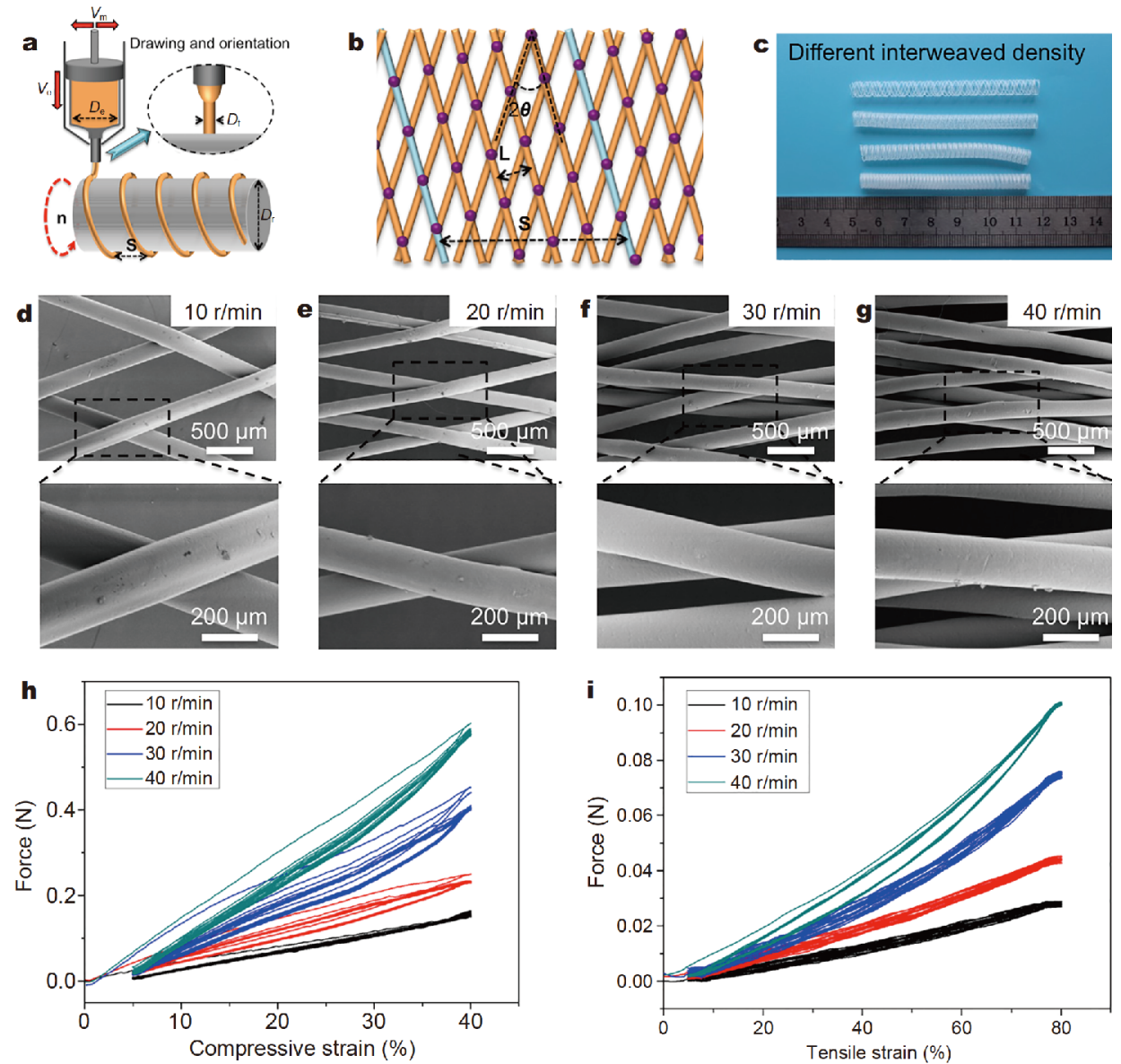

Figure 3 Microstructures and mechanical properties of PCL tubular scaffolds. Schematic models of printing process (a) and interweaved fibrousnetwork structure (b); photographs of tubular scaffolds with various microstructures (c); SEM images of crisscrossed fibers with different densities (dg); cyclic compressive (h) and tensile (i) tests for 10 cycles of 3D printed PCL tubular scaffolds. 
diverse tubular scaffolds with different microstructures (Fig. 3c).

SEM images showed that crisscrossed fibers had smooth surface and homogeneous intersections (Fig. 3dg). By theoretical calculation and analysis, the structural characteristic parameters of tubular scaffolds were determined according to the following equations:

$D_{\mathrm{f}}=\frac{D_{\mathrm{e}} \sqrt{60 V_{\mathrm{e}}}}{\sqrt[4]{n^{2} \pi^{2} D_{\mathrm{r}}^{2}+3600 V_{\mathrm{m}}^{2}}}$,

$S=\frac{60 V_{\mathrm{m}}}{n}$,

$L=\frac{60 \pi D_{\mathrm{r}} V_{\mathrm{m}}}{\sqrt{n^{2} \pi^{2} D_{\mathrm{r}}^{2}+3600 V_{\mathrm{m}}^{2}}}$,

$\theta=\arctan \frac{60 V_{\mathrm{m}}}{n \pi D_{\mathrm{r}}}$,

where $S$ is the resultant thread pitch, $L$ is the distance of adjacent fibers, $D_{\mathrm{f}}$ is the fiber diameter, $2 \theta$ is a cross angle of interlaced fibers, $D_{\mathrm{e}}$ is the inner cavity diameter of printer, $V_{\mathrm{e}}$ is extrusion flow rate, $V_{\mathrm{m}}$ is the moving velocity of the extrusion nozzle, $D_{\mathrm{r}}$ is receiver diameter, $n$ is rotational rate of receiver. These relationships were derived from the previously described model and allowed the generation of tubular scaffold in predefined and reproducible morphology. The thread pitch (Fig. 4a) and the fiber distance (Fig. $4 \mathrm{~b}$ ) were in inverse ratio to the rotational rate. The relationship between fiber diameter and rotational rate agreed with a power function of theoretical calculation model (Fig. 4c). The fiber angle of interweaved fibrous-network was inversely proportional to the rotational rate (Fig. 4d). These results demonstrated that the morphology of tubular scaffolds could be well controlled by theoretical model. Although PCL is a typical plastic material, the scaffolds exhibited superior elasticity and fatigue resistance with negligible hysteresis under dynamic deformation. Furthermore, the cyclic compressive and tensile tests demonstrated their mechanical property could be efficiently tuned by controlling the meshwork architecture (Fig. 3h, i).

\section{4-Axis printing hydrogel scaffold and PGS bio-spring}

To demonstrate the flexibility and generality of this approach, we further investigated the 4 -axis printing of alginate/polyacrylamide hydrogel (Fig. 5a-c), and a widely used thermoset biomaterial PGS [30,31]. As a representative bioelastomer, PGS can mimic the mechanical properties of soft tissues, and sustain and recover from various deformations without mechanical irritations to the surrounding tissues when implanted in a mechanically dynamic environment in the body $[32,33]$. However, it is difficult to process thermo-cured thermoset by directly 3D printing [34]. Very recently, we previously presented a general strategy to $3 \mathrm{D}$ printing thermosets
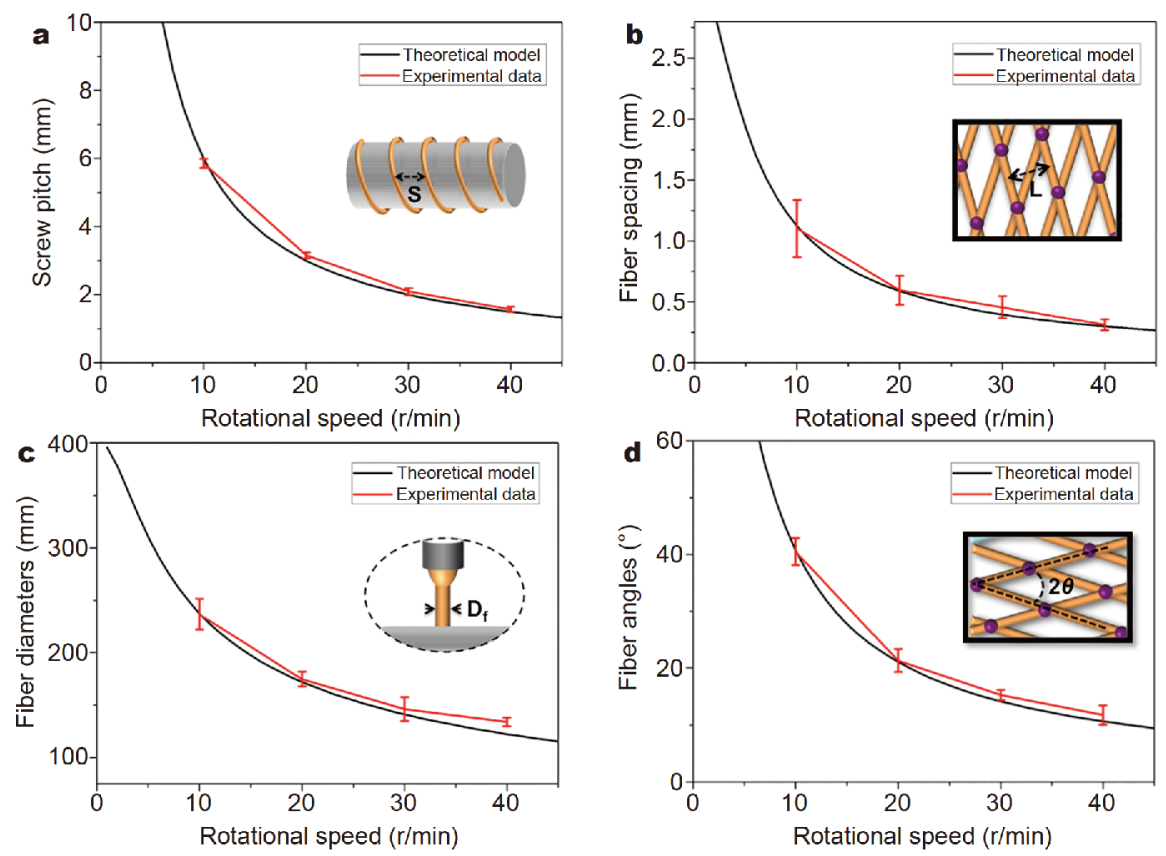

Figure 4 Predictable and reproducible printing with controllable structural parameters. The relation curves of screw pitch (a), fiber spacing (b), diameters (c) and fabric angles (d) with rotational speed. 
such as polyurethane and epoxy resin [35]. By combining with this method, we further fabricated the PGS into the bio-spring (Fig. $5 \mathrm{~d}$ ). The bio-spring showed flexibility to match the compliance with the native tubular tissues and organs (Fig. 5e). Furthermore, due to the good elasticity and anti-fatigue property of meshwork architecture, the PGS bio-spring could well resist multiple compression deformations (Fig. S2), which would enable the scaffolds to withstand stress and retain their original tubular structure during applications. As designed, the 3D printed PGS bio-spring showed well-organized hierarchical structures including the primary tubular structure, the secondary fibrous network and tertiary micropores (Fig. 5f-h and Fig. S3). The fibers were firmly stacked and fused to form a stable construct. Particle leaching method produced abundant interconnected micropores (dia- meter $=20.20 \pm 6.94 \mu \mathrm{m})$ throughout the fibers resulting in high specific surface area. The pore sizes were determined by the salt particle sizes.

\section{PGS/gelatin hybrid tubular scaffold}

To demonstrate the potential application of the 4-axis printing technology, we fabricated a hybrid tubular tissue engineered scaffold and verified its efficiency on engineering tubular cartilage both in vitro and in vivo. PGS bio-spring was used as an interior support construct for the hybrid tubular scaffold (Fig. 6a, b). Gelatin was electrospun into nanofibers and readily deposited in both the interstitial space and the inner spring for outer layer (Fig. 6c). Electrospinning is an efficient technique to produce nanofibrous construct, which mimics the natural matrix to accelerate cell adhesive growth [36,37]. The
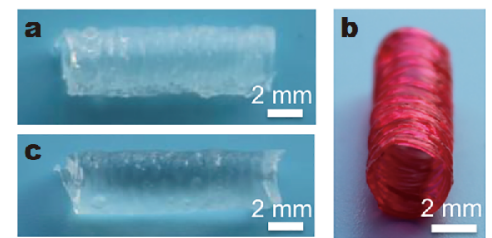

Hydrogel tubular scaffold
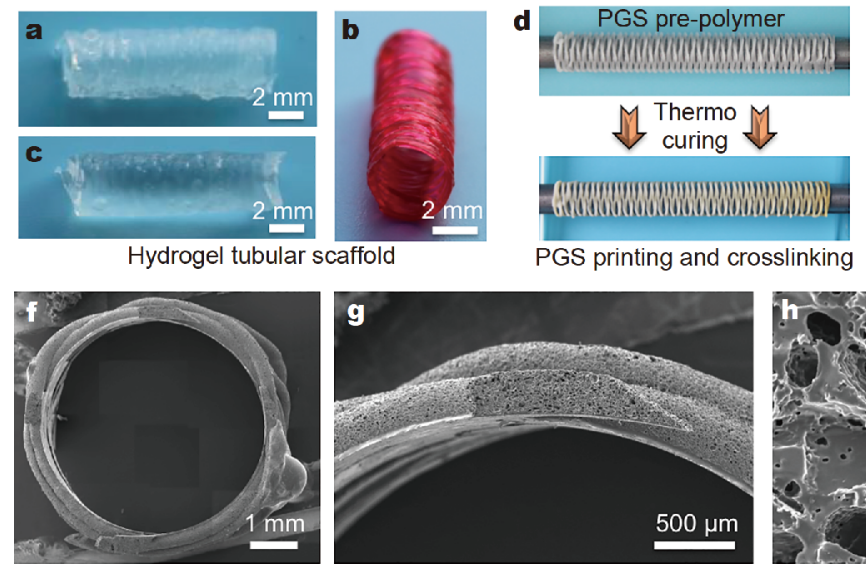

PGS printing and crosslinking

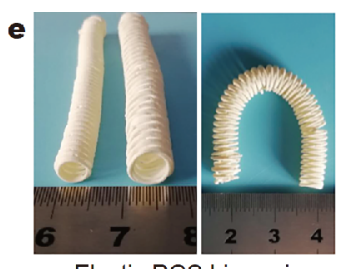

Elastic PGS bio-spring

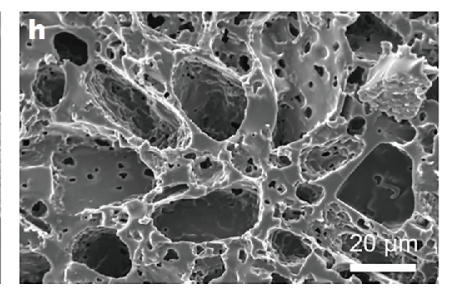

Figure 5 Versatility of the 4-axis printing strategy to fabricate hydrogel scaffold and porous thermoset bio-spring. Photographs of alginate/polyacrylamide tubular hydrogel scaffold ((a) top view; (b) visualized with red dye; (c) hydrogel scaffold filled with water); printing and crosslinking of PGS bio-spring (d); flexible and elastic PGS bio-spring (e); SEMs of PGS bio-spring with hierarchical structure in section view ((f) tube architecture; (g) woven fibers; (h) micropore structure).
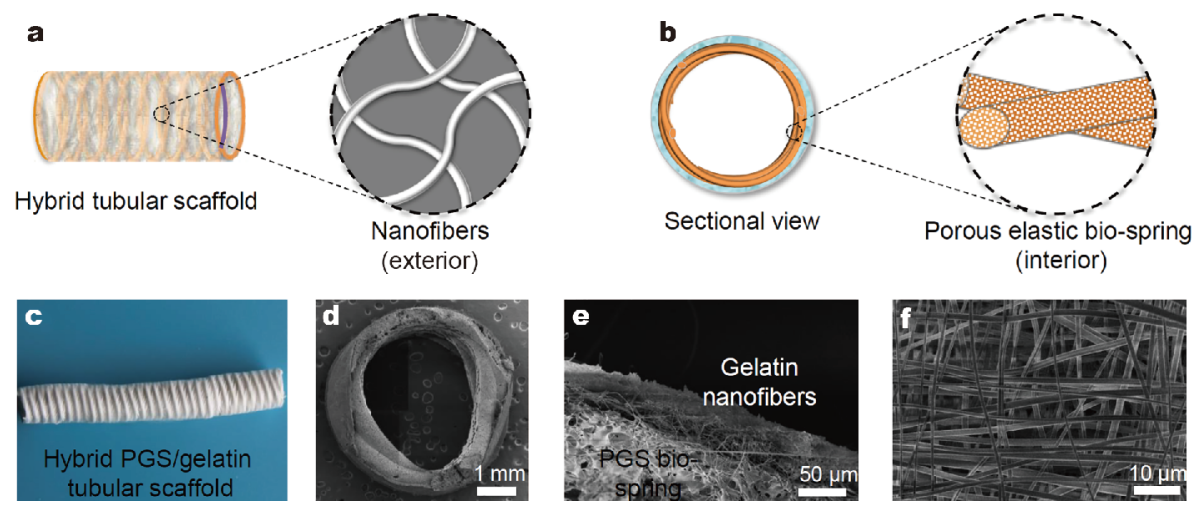

Figure 6 Upgradeable bio-spring for hybrid tubular tissue engineered scaffold. Schematic of hybrid scaffold with exterior nanofibrous structure (a) and interior PGS biospring (b); optical images of hybrid PGS/gelatin tubular scaffold (c); SEM images of scaffold with tubular structure (d), bilayer structure (e), and nanofibrous external surface (f). 
intact exterior electrospun fibrous layer was a relatively closed environment that allowed the transportation of fluids and air through the lumen (Fig. 6d-f).

\section{Tubular cartilage reconstruction}

The hybrid tubular scaffold was first evaluated for its biocompatibility. After cell seeding (Fig. 7a, b), fluorescence micrographs of live/dead and apoptosis staining showed that chondrocytes were able to effectively proliferate on the hybrid scaffold, indicating good biocompatibility and low apoptosis (Fig. $7 \mathrm{~d}-\mathrm{g}$ ). Consistent with fluorescence micrographs, DNA contents also confirmed that no statistical difference was observed between the hybrid scaffold and control group ( $p>0.05)$ (Fig. 7c), indicating that the hybrid scaffold may be an ideal scaffold for chondrocyte proliferation.

The hybrid tubular scaffold was then evaluated for its ability to engineer tubular cartilage in vitro (Fig. 8). After cultured in vitro for 8 weeks, the hybrid tubular scaffold formed cartilage-like tissue (Fig. 8a, b). Histological examinations showed that chondrocytes proliferated on the scaffold surface and secreted cartilage ECM to form tubular cartilage (Fig. 8c), as indicated by positive staining of GAG (Fig. 8d), collagen fiber (Fig. 8e) and collagen II (Fig. 8f). The result indicated that the hybrid tubular scaffold was suitable for proliferation and differentiation of chondrocytes. The satisfactory cartilage formation was related to the porous nanofibrous construct, which mimicked the natural matrix to accelerate cell adhesive growth and nutrition permeation, thus facilitating homogenous distribution of cartilage. Collectively, these results demonstrated that the hybrid tubular scaffold represented a satisfactory scaffold for tubular cartilage regeneration in vitro.

The feasibility of the hybrid tubular scaffold for in vivo cartilage regeneration was further explored in nude mice. After 12 weeks of subcutaneous implantation, the samples formed mature neocartilage with enough strength and elasticity to maintain the tubular shape (Fig. 9a-f). Histological examinations revealed that the tubular samples displayed a structure composed of connective tissue, neocartilage and a little bit of residual scaffold (Fig. 9c-f). Importantly, quantitative analyses showed that the engineered tubular cartilage had higher wet weight, thickness, and DNA content compared to normal tracheal cartilage. Moreover, Young's modulus, GAG content, and total collagen content of the engineered tubular cartilage achieved a level over $70 \%$ of normal tracheal cartilage (Fig. 9g-l). Cartilage regeneration in vivo with a specific thickness and mechanical strength was the key to long-

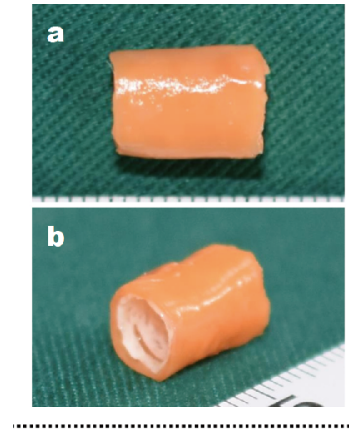

1 day
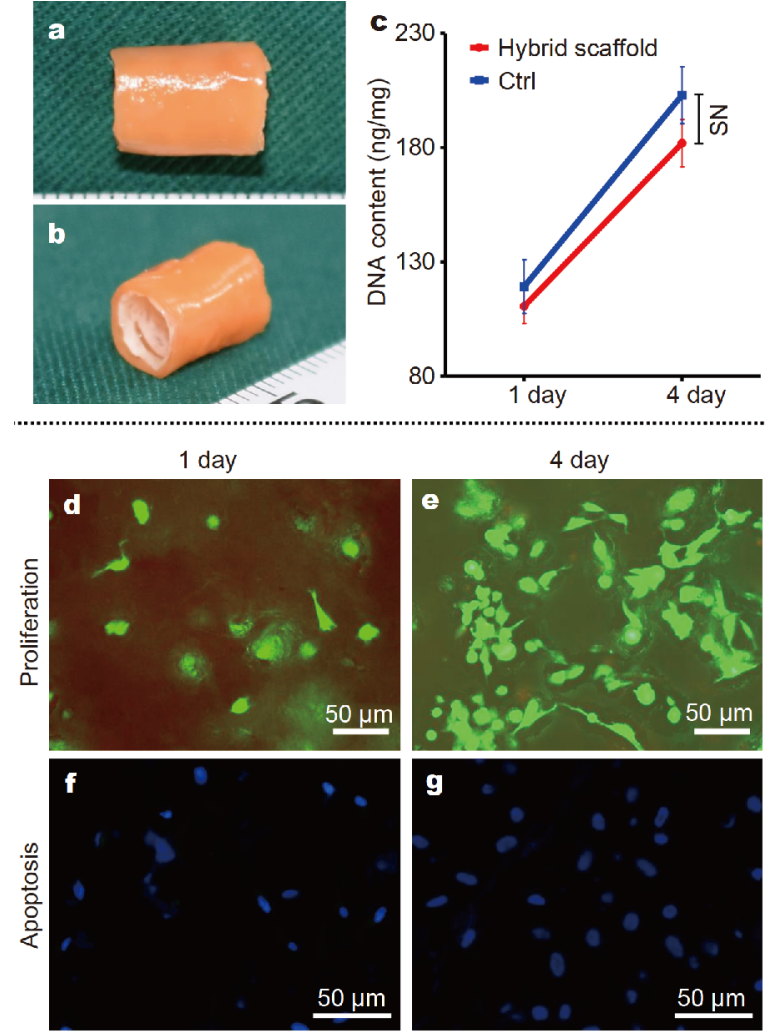

4 day

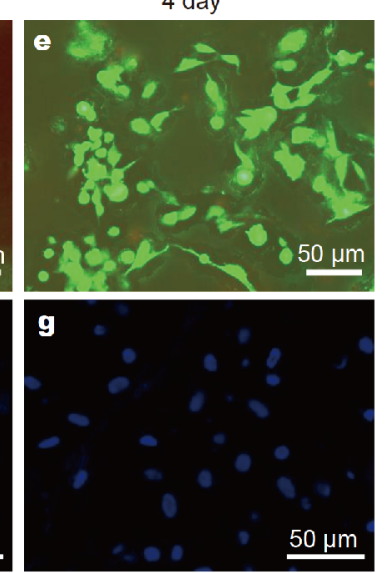

Figure 7 Biocompatibility of the hybrid scaffold. Engineered tubular cartilage constructs $(\mathrm{a}, \mathrm{b})$; no obvious difference in cell proliferation was found between the hybrid scaffold and the control group (culture medium only) (c); after cell seeding, live \& dead staining shows that cells proliferated from $1 \mathrm{~d}(\mathrm{~d})$ to $4 \mathrm{~d}$ (e) of in vitro culture; almost no apoptosis was observed from $1 \mathrm{~d}(\mathrm{f})$ to $4 \mathrm{~d}$ (g) of in vitro culture. Nuclear was stained by blue color, while apoptosis cell was stained by green color.
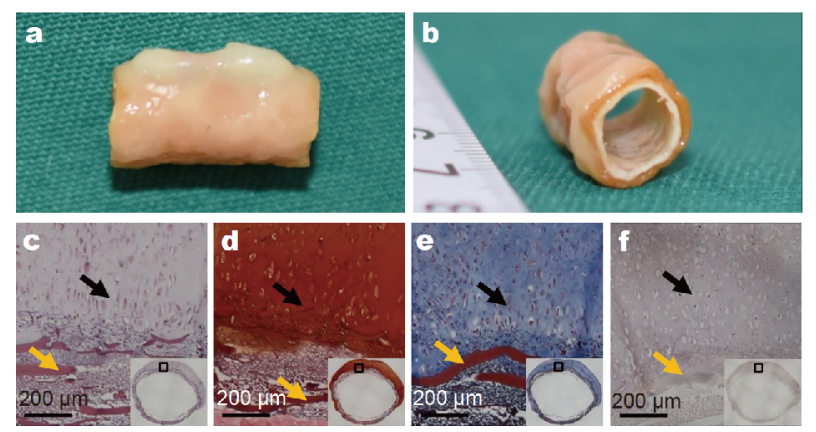

Figure 8 In vitro engineered tubular cartilage. After cell seeding, samples at 8 weeks retain their original tubular shape and form cartilage-like tissues with a matured cartilage appearance $(\mathrm{a}, \mathrm{b})$; as shown in the histological analysis of HE (c), safranin-O (d), Masson's trichrome (e) and type II collagen ( $\mathrm{f}$ ), the engineered cartilage presented typical lacunae structures and cartilage-specific ECM deposition accompanied by gradual degradation of the hybrid scaffolds. Yellow arrows indicate residual scaffold; black arrows indicate mature cartilage. 
term functional maintenance of tissue-engineered trachea [38-40]. Obviously, the ECM in the neocartilage was produced by the seeded cells, suggesting that the hybrid scaffold could provide a biomimetic microenvironment for the chondrocytes to survive. Satisfactory cartilage regeneration in vivo may benefit from good biocompatibility of the scaffold and porous nanofibrous structure produced by electrospinning, which is suitable for cell proliferation and improving nutrition and metabolite transportation. Regardless, these results indicated that the hybrid tubular scaffold represented a favourable scaffold for tubular cartilage regeneration in vivo.

\section{CONCLUSIONS}

In summary, we established a simple 4-axis printing technology to rapidly fabricate and tailor tubular constructs with diverse macroscopic geometries and controllable porous fiber weaving structures in micrometer
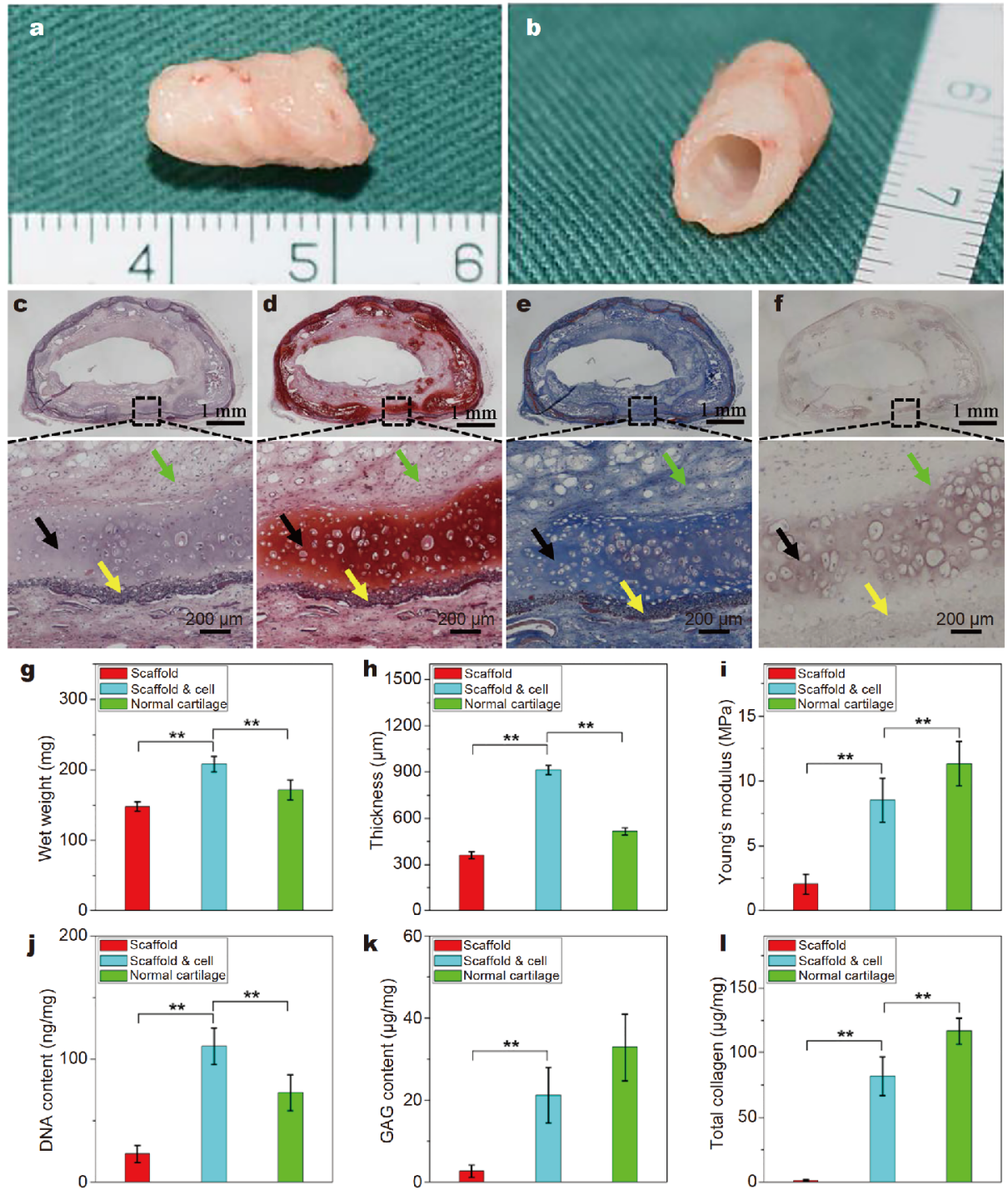

Figure 9 In vivo tubular cartilage regeneration. After 2 weeks of cultivation and 12 weeks of in vivo implantation, samples successfully regenerate relatively homogeneous mature tubular cartilage with typical lacunae structures (a, b) and cartilage-specific ECM deposition ((c) HE; (d) safranin-O; (e) Masson's trichrome; (f) type II collagen); the quantitative analyses showed that the hybrid scaffold with chondrocyte group (scaffold \& cells) achieved the highest indexes of wet weight $(\mathrm{g})$, thickness $(\mathrm{h})$, and DNA content (j) with significant differences among groups; as for the quantitative indexes of Young's modulus (i), GAG content (k), and total collagen content (l), the scaffold \& cells group are still significantly higher compared with scaffold only group (without cells), but slightly lower than fresh normal trachea cartilage group. Green arrow indicates normal connective tissue; black arrow indicates neocartilage; yellow arrow indicates residual scaffold. Statistically significance: ${ }^{* *} p<0.01$. 
scale. This printing method is highly versatile and can be readily applied to diverse materials. We believe that this technology provides a powerful way to fabricate tissue engineered scaffolds for a wide range of tubular tissues, the utilization of which was demonstrated to efficiently engineer trachea-like cartilage tissue. Furthermore, this technology can also be used for other biomedical applications such as vascular inner stents and external support.

Received 23 June 2019; accepted 26 July 2019;

published online 6 September 2019

1 Yu P, Clayman GL, Walsh GL. Long-term outcomes of microsurgical reconstruction for large tracheal defects. Cancer, 2011, 117: 802-808

2 Goh CSL, Joethy JV, Tan BK, et al. Large animal models for longsegment tracheal reconstruction: a systematic review. J Surg Res, 2018, 231: 140-153

3 Virk JS, Zhang H, Nouraei R, et al. Prosthetic reconstruction of the trachea: a historical perspective. World J Clin Cases, 2017, 5: 128133

4 Gong W, Lei D, Li S, et al. Hybrid small-diameter vascular grafts: anti-expansion effect of electrospun poly $\varepsilon$-caprolactone on heparin-coated decellularized matrices. Biomaterials, 2016, 76: 359370

5 Dikina AD, Alt DS, Herberg S, et al. A modular strategy to engineer complex tissues and organs. Adv Sci, 2018, 5: 1700402

6 Hussey GS, Keane TJ, Badylak SF. The extracellular matrix of the gastrointestinal tract: a regenerative medicine platform. Nat Rev Gastroenterol Hepatol, 2017, 14: 540-552

7 de Jonge PKJD, Simaioforidis V, Geutjes PJ, et al. Recent advances in ureteral tissue engineering. Curr Urol Rep, 2015, 16: 465

8 Del Gaudio C, Baiguera S, Ajalloueian F, et al. Are synthetic scaffolds suitable for the development of clinical tissue-engineered tubular organs? J Biomed Mater Res, 2014, 102: 2427-2447

9 Góra A, Pliszka D, Mukherjee S, et al. Tubular tissues and organs of human body-challenges in regenerative medicine. J Nanosci Nanotechnol, 2016, 16: 19-39

10 Versteegden LR, van Kampen KA, Janke HP, et al. Tubular collagen scaffolds with radial elasticity for hollow organ regeneration. Acta Biomater, 2017, 52: 1-8

11 Janke HP, Bohlin J, Lomme RMLM, et al. Bioinspired coupled helical coils for soft tissue engineering of tubular structures-improved mechanical behavior of tubular collagen type I templates. Acta Biomater, 2017, 59: 234-242

12 Kang H, Zhang K, Pan Q, et al. Remote control of intracellular calcium using upconversion nanotransducers regulates stem cell differentiation in vivo. Adv Funct Mater, 2018, 28: 1802642

13 Park JH, Hong JM, Ju YM, et al. A novel tissue-engineered trachea with a mechanical behavior similar to native trachea. Biomaterials, 2015, 62: 106-115

14 Park JH, Park JY, Nam IC, et al. A rational tissue engineering strategy based on three-dimensional (3D) printing for extensive circumferential tracheal reconstruction. Biomaterials, 2018, 185: 276-283

$15 \mathrm{Wu}$ W, Allen RA, Wang Y. Fast-degrading elastomer enables rapid remodeling of a cell-free synthetic graft into a neoartery. Nat Med, 2012, 18: 1148-1153
16 Huang X, Jiao T, Liu Q, et al. Hierarchical electrospun nanofibers treated by solvent vapor annealing as air filtration mat for highefficiency PM2.5 capture. Sci China Mater, 2018, 62: 423-436

17 Lou L, Kong X, Zhu T, et al. Facile fabrication of interconnectedmesoporous $\mathrm{T}-\mathrm{Nb}_{2} \mathrm{O}_{5}$ nanofibers as anodes for lithium-ion batteries. Sci China Mater, 2019, 62: 465-473

18 Lei D, Yang Y, Liu Z, et al. 3D printing of biomimetic vasculature for tissue regeneration. Mater Horiz, 2019, 6: 1197-1206

19 Robertson ID, Yourdkhani M, Centellas PJ, et al. Rapid energyefficient manufacturing of polymers and composites via frontal polymerization. Nature, 2018, 557: 223-227

20 Gantenbein S, Masania K, Woigk W, et al. Three-dimensional printing of hierarchical liquid-crystal-polymer structures. Nature, 2018, 561: 226-230

21 Nune KC, Li S, Misra RDK. Advancements in three-dimensional titanium alloy mesh scaffolds fabricated by electron beam melting for biomedical devices: mechanical and biological aspects. Sci China Mater, 2018, 61: 455-474

22 Correa VL, Garza KM, Murr LE. Vascularization in interconnected 3D printed Ti-6Al-4V foams with hydrogel matrix for biomedical bone replacement implants. Sci China Mater, 2018, 61: 565-578

23 Kang HW, Lee SJ, Ko IK, et al. A 3D bioprinting system to produce human-scale tissue constructs with structural integrity. Nat Biotechnol, 2016, 34: 312-319

24 Gao F, Xu Z, Liang Q, et al. Direct 3D printing of high strength biohybrid gradient hydrogel scaffolds for efficient repair of osteochondral defect. Adv Funct Mater, 2018, 28: 1706644

25 Itoh M, Nakayama K, Noguchi R, et al. Scaffold-free tubular tissues created by a bio-3D printer undergo remodeling and endothelialization when implanted in rat aortae. PLoS ONE, 2015, 10: e0136681

26 Wang Y, Ameer GA, Sheppard BJ, et al. A tough biodegradable elastomer. Nat Biotechnol, 2002, 20: 602-606

$27 \mathrm{Xu} \mathrm{Y,} \mathrm{Li} \mathrm{D,} \mathrm{Yin} \mathrm{Z,} \mathrm{et} \mathrm{al.} \mathrm{Tissue-engineered} \mathrm{trachea} \mathrm{regeneration}$ using decellularized trachea matrix treated with laser micropore technique. Acta Biomater, 2017, 58: 113-121

28 Yan D, Zhou G, Zhou X, et al. The impact of low levels of collagen IX and pyridinoline on the mechanical properties of in vitro engineered cartilage. Biomaterials, 2009, 30: 814-821

29 Chen J, Yuan Z, Liu Y, et al. Improvement of in vitro threedimensional cartilage regeneration by a novel hydrostatic pressure bioreactor. Stem Cell Transl Med, 2017, 6: 982-991

30 Mahdavi A, Ferreira L, Sundback C, et al. A biodegradable and biocompatible gecko-inspired tissue adhesive. Proc Natl Acad Sci USA, 2008, 105: 2307-2312

31 Ye X, Lu L, Kolewe ME, et al. Scalable units for building cardiac tissue. Adv Mater, 2014, 26: 7202-7208

32 Rai R, Tallawi M, Grigore A, et al. Synthesis, properties and biomedical applications of poly(glycerol sebacate) (PGS): a review. Prog Polymer Sci, 2012, 37: 1051-1078

33 Loh XJ, Abdul Karim A, Owh C. Poly(glycerol sebacate) biomaterial: synthesis and biomedical applications. J Mater Chem B, 2015, 3: 7641-7652

34 Yeh YC, Highley CB, Ouyang L, et al. 3D Printing of photocurable poly(glycerol sebacate) elastomers. Biofabrication, 2016, 8: 045004

35 Lei D, Yang Y, Liu Z, et al. A general strategy of 3D printing thermosets for diverse applications. Mater Horiz, 2019, 6: 394-404

36 Mo X, Li D, Ei-Hamshary HA, et al. Electrospun nanofibers for tissue engineering. J Fiber Bioeng Inform, 2013, 6: 225-235

37 Yang JM, Yang JH, Tsou SC, et al. Cell proliferation on PVA/ 
sodium alginate and PVA/poly( $\gamma$-glutamic acid) electrospun fiber. Mater Sci Eng-C, 2016, 66: 170-177

38 Kojima K, Vacanti CA. Tissue engineering in the trachea. Anat Rec, 2014, 297: 44-50

39 Gong YY, Xue JX, Zhang WJ, et al. A sandwich model for engineering cartilage with acellular cartilage sheets and chondrocytes. Biomaterials, 2011, 32: 2265-2273

40 Tanaka H, Maeda K, Okita Y. Transplantation of the cryopreserved tracheal allograft in growing rabbits. J Pediatr Surg, 2003, 38: $1707-1711$

Acknowledgements This work was supported by the National Key Research and Development Program of China (2018YFB1105602 and 2017YFC1103900), the National Natural Science Foundation of China (21574019, 81320108010, 81571823 and 81871502), the Natural Science Foundation of Shanghai (18ZR1401900), the Fundamental Research Funds for the Central Universities, DHU Distinguished Young Professor Program (LZA2019001), the Science and Technology Commission of Shanghai (17DZ2260100 and 15DZ1941600), the Program for Shanghai Outstanding Medical Academic Leader, and the Program of Shanghai Technology Research Leader.

Author contributions Lei D designed and performed all experiments, analyzed the data and prepared the manuscript. Xu Y performed the chondrocytes culture and trachea reconstruction. Luo B and Shen A contributed to 3D printing. Guo Y performed SEM characterization and statistic analysis. Liu Z and Wang S performed PGS polymer synthesis. Wang D and Yang $\mathrm{H}$ performed the manufacture of receivers. Xuan $\mathrm{H}$ contributed to electrospinning. Zhang $\mathrm{Y}$ contributed to mechanical testing. He C and Qing FL contributed to the discussion and analysis of the experimental result. Zhou G discussed the concept and contributed to the writing of the manuscript. You $\mathrm{Z}$ supervised the whole work and wrote the manuscript.

Conflict of interest The authors declare no conflict of interest.

Supplementary information Supporting data are available in the online version of the paper.

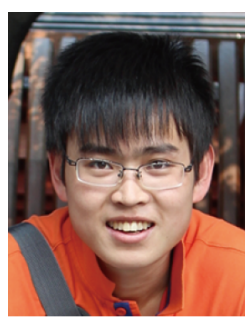

Dong Lei is a PhD student at the State Key Laboratory for Modification of Chemical Fibers and Polymer Materials at Donghua University. From 2013 to now, he has been conducting his master and doctoral research at Donghua University. His current research involves biomaterials, 3D printing and tissue regeneration.

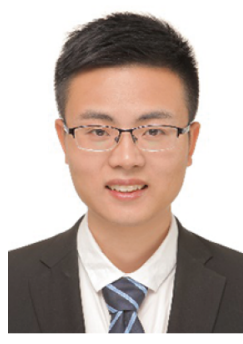

Yong $\mathrm{Xu}$ received his MCs degree from the Department of Thoracic Surgery, Shanghai Pulmonary Hospital, Tongji University School of Medicine in 2018. Now he is continuing to study for a $\mathrm{PhD}$ degree in the Department of Thoracic Surgery, Shanghai Pulmonary Hospital, Tongji University School of Medicine. His research interests focus on cartilage regenerative biomaterials and functional tracheal reconstruction.

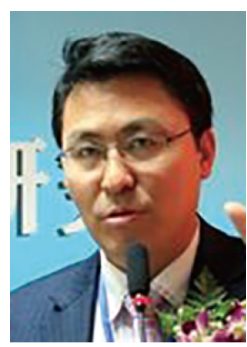

Guangdong Zhou is a professor of Medical College of Shanghai Jiao Tong University, Executive Vice-Director of National Center for Tissue Engineering Research, Former SecretaryGeneral and Executive Director of Tissue Engineering and Regenerative Medicine Branch of Chinese Society of Biomedical Engineering. His research interests focus on the functional cartilage regeneration and clinical application transformation, construction of cartilage in vitro and precise regulation of its three-dimensional morphology (auricle, trachea, etc.), and repair of various cartilage defects in large animals.

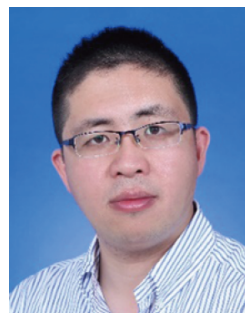

Zhengwei You is a professor and the chair of Department of Composite Materials at Donghua University. He received his BSc degree from Shanghai Jiao Tong University and $\mathrm{PhD}$ degree from Shanghai Institute of Organic Chemistry. $\mathrm{He}$ conducted his postdoctoral research at Georgia Institute of Technology and University of Pittsburgh. Prior to joining Donghua University, he was an innovation manager in Bayer Material Science. His current research involves stretchable electronics. smart polymers, biomaterials, 3D printing, and

\section{4轴打印微纤维管状支架及其气管软骨应用}

雷东 ${ }^{1,2}$, 罗彬 ${ }^{2}$, 郭一凡 ${ }^{2}$, 汪地 ${ }^{4}$, 杨浩 ${ }^{4}$, 王少飞 ${ }^{2}$, 轩慧霞 ${ }^{2}$, 沈傲 ${ }^{2}$, 张轶 ${ }^{2}$, 刘增贺 ${ }^{1,2}$, 何创龙 ${ }^{1}$, 卿风领 ${ }^{1}$, 徐勇 ${ }^{3 *}$, 周广东 ${ }^{5,6^{*}}$, 游正伟 ${ }^{1,2^{*}}$

摘要 管状组织大段缺损的重建是临床治疗上的一个难题. 如何设 计并制备具有理想结构的功能管状支架用于管状组织修复仍然是 再生医学中的一个主要挑战. 本研究采用4轴打印系统快速地制备 了具有多级结构的管状组织工程支架, 该方法适用于多种生物材 料, 如水凝胶、热塑性生物材料以及热固性生物材料. 以聚已内酯 (PCL)为例, 我们成功制备了管状结构可调、类编织网格结构可控, 径向弹性、轴向柔性及管腔开放性良好的支架. 作为管状组织再 生的初步应用, 我们利用 4 轴打印技术制备了聚癸二酸甘油酯 (PGS)的热固性生物弹簧, 并与静电纺技术相结合制备了杂化的 $\mathrm{PGS} /$ 明胶纳米纤维管状支架. 该支架接种软骨细胞后在体外培养 和裸鼠皮下植入均可形成成熟的管状软骨组织, 有望应用于气管 软骨重建. 